\title{
RITMO Y VARIACIÓN DE LOS REFRANES: DIACRONÍA, DIATOPÍA Y MANIPULACIONES
}

\author{
RHYTHM AND VARIATION OF PROVERBS: \\ DIACHRONY, GEOGRAPHICAL VARIATION \\ AND MODIFIED PROVERBS
}

\section{RYTHME ET VARIATION DES PROVERBES: DIACHRONIE, DIATOPIE ET DÉTOURNEMENTS}

\author{
Alexandra Oddo \\ Université Paris Nanterre
}

\begin{abstract}
Resumen: La importancia del metro, del ritmo y de la rima en los refranes ha sido señalada en repetidas ocasiones y se verifica en numerosos ejemplos que, para su observación, se han limitado hasta ahora al estudio de un refrán «fijado» en un estado de lengua sincrónico, sin tener en cuenta su potencial variabilidad tanto en diacronía como en diatopía. Este trabajo pretende investigar la permanencia de dichas características cuando los enunciados se ven sometidos a transformaciones que se mantienen en diacronía o en diatopía, o incluso cuando son manipulados voluntariamente.
\end{abstract}

Palabras clave: refranes, ritmo, variación diacrónica, variación diatópica. 
Abstract: The importance of metrics, rhythm and rhyme in proverbs has been noticed and pointed out in many cases, but the studies have so far been limited to a fixed vision of the saying. A diachronic and a geographical approach show that every kind of variation can produce variants and this work aims at investigating the permanence of these remarkable characteristics when the statements are transformed.

Keywords: proverbs, rhythm, diachrony, geographical variation.

Résumé: L'importance de la métrique, du rythme et de la rime au sein des proverbes a été signalée à plusieurs reprises. Elle est remarquable dans de nombreux exemples qui présentent tous un proverbe figé dans un état de langue synchronique qui ne tient donc pas compte de ses possibilités de variation dans le temps (diachronie) et dans l'espace (diatopie). L'objectif de ce travail est de mesurer l'importance de ces caractéristiques rythmiques - et leur éventuelle conservation ou élimination lorsqu'il y a variation, transformation ou manipulation.

Mots-clés: proverbes, rythme, variation diachronique, variation diatopique. 
a importancia del ritmo en los refranes ha sido señalada en repetidas ocasiones tanto por los paremiólogos ${ }^{1}$ como por investigadores especialistas de la métrica y del ritmo. Así, pudo observar Arcadio Pardo que los metricistas a menudo habían podido considerar que muchos refranes tradicionales eran versos $^{2}$ y más de una vez se ha postulado la existencia de un artificio rítmico propio de estas producciones ${ }^{3}$, situándose su especificidad entre verso y prosa: «[...] estos refranes están entre el verso y la prosa, y poseen su propia entidad rítmica, que todos han reconocido en la lengua española» ${ }^{4}$.

La presencia de un patrón rítmico estructurante es una hipótesis que se ha verificado con numerosos ejemplos que, hasta ahora, se han limitado al estudio de un refrán «fijado» en un estado de lengua sincrónico y sin tener en cuenta su potencial variabilidad tanto en diacronía como en diatopía. Queda, pues, por investigar si dicha característica y dicho patrón rítmico se conservan a pesar de las transformaciones que pueden en varias situaciones discursivas alterar

1 Se pueden citar como referencias recientes los trabajos de Anscombre, Jean-Claude, «Estructura(s) métrica(s) en los refranes», Paremia, 8 (1999), pp. 25-36; «Matrices rythmiques et parémies», en Jean-Claude Anscombre, Bernard Darbord y Alexandra Oddo (coords.), La parole exemplaire. Introduction à une étude linguistique des proverbes, Paris, Armand Colin, 2012, pp. 147-158; DARBORD, Bernard, «La rhétorique du proverbe», en Jean-Claude Anscombre, Bernard Darbord y Alexandra Oddo (coords.), La parole exemplaire..., cit., pp. 170-182; D’ANDREA, Giulia, "Qui dit proverbe... dit rythme ?», Scolia, 31 (2017), pp. 101-118 y VILliERS, Damien, «Marqueurs stylistiques: leur poids dans la définition et la genèse des proverbes», Scolia, 31 (2017), pp. 79-100 pero el interés que suscita esta relación empieza ya en la década de los ochenta. Véase RoDEGEM, François, «La parole proverbiale», en François Suard y Claude Buridant (coords.), Richesse du proverbe, Lille, Presses Universitaires, 1984, pp. 121-135.

2 PARdo, Arcadio, "Verso aislado, verso solo, verso-poema», Rhythmica, II, 2 (2004), pp. 201-233, p. 203.

3 «El refrán tiene normalmente algún artificio rítmico que lo acerca al verso» (DomíNguez Caparrós, José, Diccionario de métrica española, Madrid, Paraninfo, Colección filológica, 1985, p. 123).

${ }^{4}$ López Estrada, Francisco, Métrica española del siglo XX, Madrid, Editorial Gredos, 1969, pp. 86-87. 
el refrán, dándose incluso el caso de modificaciones perennes. Los ejemplos siguientes ponen de manifiesto la transformación que pueden experimentar los refranes ${ }^{5}$ en diacronía:

(1) Haz bien y no cates a quien (siglo Xv - García de Castro ${ }^{6}$, Santillana ${ }^{7}$ ) $>$ Haz bien y no mires a quién ( siglo $\mathrm{xx}^{8}$ ).

(2) Quien burla al burlador cien días gana de perdón (siglo XV - García de Castro, Santillana) $>$ Quien roba a un ladrón, tiene cien años de perdón (siglo XX - Doval, Junceda).

Nuestro estudio quiere abarcar también la posibilidad de una variación diatópica que queda plasmada en los dos ejemplos siguientes:

(3) A falta de pan buenas son tortas (España, Doval) $>$ A falta de pan, tortillas (cemitas /cemas / semas / acemitas / casabe) .

(4) A caballo presentado, no hay que mirarle el diente / A caballo regalado, no le mires el dentado (España, Doval) / A caballo regalado, no le mires el diente (España, Junceda) $>$ A caballo regalado (no se le mira el pelo / el colmillo / no se le busca colmillo) ${ }^{10}$.

Y, por fin, incluye también las distintas manipulaciones a las que pueden verse sometidos los refranes, por ser enunciados compartidos por una comunidad lingüística y, por ende, estar más expuestos al principio de econimia descrito por Pottier ${ }^{11}$.

(5) A la cama no te irás sin saber una cosa más > A la cama no te irás, sin deber un poco más.

${ }^{5}$ Los refraneros que constituyen el corpus de este trabajo se citarán en este trabajo por el nombre de su autor.

${ }^{6}$ García de Castro, Diego, Seniloquium (ca. 1480), traducción y edición crítica de Fernando Cantalapiedra Erostarbe y Juan Moreno Uclés, Valencia, Publicacions de la Universitat de València, 2006.

${ }^{7}$ Marqués de Santillana (Íñigo López de Mendoza), Refranes que dizen las viejas tras el fuego (1454), edición de Hugo Bizzarri, Kassel, Reichenberger, 1995.

8 Las dos colecciones contemporáneas son las de Doval, Gregorio, Refranero temático español, Madrid, Ediciones del Prado, 1997 y JuncedA, Luis, Diccionario de refranes, dichos y proverbios, Madrid, Espasa-Calpe, 1998.

9 Estas variantes se registran en varios países latinoamericanos. Véase SEvilla MuÑoz, Julia, y Cantera Ortiz De Urbina, Jesús (dirs.), 1001 refranes españoles con su correspondencia en ocho lenguas (alemán, árabe, francés, inglés, italiano, polaco, provenzal y ruso), Madrid, Eunsa, 2. ${ }^{a}$ edición revisada y ampliada, 2008, p. 36.

${ }^{10}$ Ibid., pp. 31-32.

11 Este concepto lo desarrolla Bernard PotтiER en Semántica general, Madrid, Gredos, 1993, p. 20: «El que una novela contemporánea tenga por título Érase dos veces implica el conocimiento de una lexía de referencia: la fórmula del pasado mítico érase una vez. El título funciona como ecónimo de la lexía memorizada». 
(6) Más vale pájaro en mano que ciento volando > Más vale euro en la mano, que ciento volando ${ }^{12}$.

\section{Sobre variación y ritmo. Conceptos y terminología}

El concepto de variación, así como el de ritmo, requieren que se puntualice el enfoque teórico que constituye el marco de esta reflexión mediante un breve recorrido a través de la terminología usual y las definiciones que se manejan en estos dos ámbitos.

La principal característica de la lengua, cuando se materializa en el habla, es que varía, en diacronía y en sincronía, dentro de lo que llamamos un diasistema. Según el Diccionario de términos clave de ELE del Centro Virtual Cervantes:

Se entiende por variación lingüística el uso de la lengua condicionado por factores de tipo geográfico, sociocultural, contextual o histórico. La forma como los hablantes emplean una lengua no es uniforme, sino que varía según sus circunstancias personales, el tiempo y el tipo de comunicación en que están implicados. En función del factor que determina el distinto empleo de una misma lengua, se consideran varios tipos de variaciones: la variación funcional o diafásica, la variación sociocultural o diastrática, la variación geográfica o diatópica y la variación histórica o diacrónica ${ }^{13}$.

De lo dicho se infiere que la lengua estándar es solo una proyección funcional. Esta capacidad de variación es inherente a la lengua que diferencia, por definición, la norma, una construcción funcional idealizada, y el habla, sometida a variaciones de distinta índole ya que la homogeneidad y la unidad de un sistema lingüístico siempre se verán impactadas por la diversidad de los usos. En el ámbito de los refranes todas estas variaciones nos interesan porque, en mayor o menor medida, van a alterar los refranes y producir variantes tanto en diacronía, con la corrección del arcaísmo 'catar' del ejemplo (1) como en sincronía, con la documentación de variantes léxicas en diccionarios contemporáneos de refranes en el caso del ejemplo (4) 'diente', 'colmillo' y 'pelo'.

También conviene destacar que la variación conlleva implicaciones en el ámbito de la semántica ya que a la cuestión de la producción de variantes ya evocada debe añadirse la noción conexa

$\overline{12}$ «Refranes de la crisis» documentados en el sitio Foro-ciudad.com [https://www. foro-ciudad.com/asturias/serin/mensaje-11077050.html] (consultado el 01/07/2020).

13 Centro virtual Cervantes, Diccionario de términos clave de ELE, «La variación lingüística». [https://cvc.cervantes.es/ensenanza/biblioteca_ele/diccio_ele/diccionario/variacionlinguistica.htm]. 
de variable, es decir, el concepto abstracto que designa las diferentes unidades del conjunto paradigmático de lexías que pueden ser solicitadas en el habla. En efecto, las situaciones de parasinonimia descritas por Pottier ${ }^{14}$ se producen también en el marco de los refranes y pueden afectar ora su léxico ora el enunciado en su conjunto $^{15}$. Esta noción es precisamente la que permite deslindar satisfactoriamente las variantes y las manipulaciones: en los ejemplos sacados a colación en la introducción, el (3) y el (4) presentan una variación limitada a categorías bien definidas -en (3) la masa y los ingredientes que entran en su preparación y en (4) las características físicas del caballo-, mientras que los refranes de (5) y (6) han sido sometidos, al pasar de 'saber' a 'deber' $y$, de un modo aún más llamativo, de 'pájaro' a 'euro', a manipulaciones imprevisibles en el ámbito lingüístico que García-Page califica de «alteraciones estructurales de forma libre y espontánea, no reguladas o conformes a una instrucción o canon» ${ }^{16}$.

Las variaciones potenciales de los refranes que ofrece la lengua se articulan en este trabajo con otro eje de estudio, el del ritmo, concebido en este como una serie de rasgos métricos, rítmicos o rímicos ${ }^{17}$ que para muchos investigadores son una característica inherente de estos enunciados ${ }^{18}$. Cabe señalar que numerosos estudiosos del refranero han relacionado la presencia del ritmo en el refranero con el ritmo general y natural que posee la lengua que los crea. En esta vena se sitúan Taylor ${ }^{19}$, desde los años treinta, y más tarde Anscombre ${ }^{20}$, ya que según ellos, se verifica una adaptación de los patrones rítmicos de los refranes a la poesía natural de cada lengua (grupos fónicos medios, tipos de versos predilectos, sílabas tónicas, etc.). Como tuvimos ocasión de señalarlo en un

\footnotetext{
${ }_{14}$ Pottier, Bernard, Semántica general, cit. p. 38.

15 Ibid., p. 63.

16 García PAge, Mario, «Los otros ‘falsos amigos' de la fraseología: variantes genuinas y variantes espurrias», en Carmen González Royo y Pedro Mogorrón Huerta (eds.), Fraseología contrastiva: lexicografía, traducción y análisis de corpus, Alicante, Publicaciones de la universidad de Alicante, 2011, pp. 95-108, p. 96.

17 Para Villers, Damien, «Marqueurs stylistiques...», cit., p. 80, los marcadores estilísticos deben entenderse como hiperónimo que engloba todos los fenómenos formales y sonoros que confieren al enunciado un estilo distintivo.

${ }^{18}$ Ibid., pp. 82-85.

19 TAYlOR, Archer, The Proverb, Cambridge, Massachusetts, Harvard University Press y Hatboro (Pennsylvania)/Copenhagen (Denmark), Folklore Associates-Rosenkilde and Bagger, 1931.

${ }^{20}$ Anscombre, Jean-Claude, «Matrices rythmiques et parémies», cit., p. 152.
} 
trabajo anterior ${ }^{21}$, las definiciones que se aplican al corpus poético en gran medida pueden trasladarse a los refranes, aunque este planteamiento metodológico haya podido desencadenar algunas críticas por parte de especialistas de la poesía ${ }^{22}$. De hecho, el modelo de estudio del ritmo preconizado por Pardo para la poesía se puede aplicar a una gran diversidad de enunciados lingüísticos, como refranes, títulos ${ }^{23}$ o eslóganes y permite evaluar, mediante una serie de elementos estables y sistemáticos, presentes todos o solo en parte, si una secuencia dada es portadora de un ritmo.

La observación del ritmo empieza por la del metro, de las pausas, luego se adentra en el estudio primordial de la distribución de los acentos, para ahondar finalmente esta investigación con un estudio pormenorizado de las unidades rítmicas y de sus encadenamientos ${ }^{24}$.

Recordemos con Arcadio Pardo que «los metricistas han señalado que, efectivamente, pueden considerarse versos muchos de los refranes tradicionales ${ }^{25}$. Según Anscombre también, el ritmo es una característica estructurante de las paremias: «no se trata de estructuras que se fundamentan en la rima - $\mathrm{o}$ no solamente- sino de estructuras rítmicas (ya que las primeras son solo una modalidad de las segundas) ${ }^{26}$. Asimismo menciona López Estrada en Métrica española del siglo $X X$ una serie de procedimientos que le confieren al refrán «su propio artificio rítmico» y «su propia entidad rítmica» ${ }^{27}$. Estas aseveraciones incitan a considerar que dichos patrones rítmicos son un rasgo definitorio de los refranes, que el ritmo ocupa un lugar preponderante para la identificación de estos enunciados con

${ }^{21}$ ODdo, Alexandra, «Historia de una pareja: el ritmo en el refranero español», Rhythmica, Revista española de métrica comparada, 13 (2015), pp. 173-192.

22 Especialmente Gouvard, como se explica en Villers, Damien, «Marqueurs stylistiques», cit., p. 90.

${ }^{23}$ Véase PARDo, Arcadio: «Verso aislado...», cit. y en este mismo volumen «La métrica en el refranero español».

24 Pardo, Madeleine y Arcadio, Précis de métrique espagnole, Paris, Armand Colin, 2010, p. 57.

25 PARDO, Arcadio: «Verso aislado...», cit., p. 203.

26 AnsCOMBRE, Jean-Claude, "Grandeurs et misères linguistiques de la parémiologie», Crisol, 14 (2011), pp. 59-81, p. 73. Véase también Dessons, Gérard, «Pour une rythmique du proverbe», La Licorne, 125 (1984), pp. 22-33.

27 López Estrada, Francisco, Métrica española, cit., pp. 86-87. Véase también DomínGUEZ CAPARrós, José, Diccionario de métrica española, cit., p. 123: «El refrán tiene normalmente algún artificio rítmico que lo acerca al verso. El hecho de que se relacionen dos ideas lleva a que la tendencia a este paralelismo se vea reforzada por la rima o por el isosilabismo de los dos miembros». 
la categoría lingüística de los refranes ${ }^{28}$ y que, de hecho, también cumple un papel importante en los procesos de transformación de estos enunciados que conllevan tanto la evolución en diacronía y en diatopía como la desautomatización ${ }^{29}$.

\section{Ritmo y variación diacrónica}

Para medir las implicaciones de la variación diacrónica en el ritmo de los refranes, nos detendremos en tres grandes tipos de transformaciones y limitaremos nuestro corpus a algunos ejemplos sobresalientes que permiten, sin embargo, ejemplificar el sistema del refranero: la evolución estructural; la evolución morfosintáctica y los cambios léxicos.

Los cambios estructurales en diacronía pueden ser de diversa índole y conllevar una reestructuración total del enunciado. Esto se produce en el caso de los refranes truncados o alargados, o cuando se modifica la estructura de origen de la paremia ${ }^{30}$ por ejemplo. Estos ajustes se producen en otras lenguas y Anscombre menciona el proceso de lexicalización al que se ven sometidas las nuevas estructuras:

Il peut se faire - et les exemples en sont fréquents - que la forme tronquée se lexicalise et donne naissance à une nouvelle entité parémique, qui élimine généralement la forme pleine. Par exemple la forme originale A chacun son métier, et les vaches seront bien gardées est en train de disparaître au profit de la forme tronquée $A$ chacun son métier: 292 attestations pour la forme tronquée dans frTenTen 12 contre seulement 12 pour la forme pleine. D'une façon analogue en espagnol, la forme tronquée Cada loco con su tema (Spanish $\mathrm{WaC}=11$ ) est en train d'éliminer la forme complète Cada loco con su tema y cada lobo por su senda (Spanish $\mathrm{WaC}=2)^{31}$.

28 Anscombre, Jean-Claude, «Estructura(s) métrica(s) en los refranes», Paremia, 8 (1999), pp. 25-36, p. 32 y «Matrices rythmiques et parémies», cit., p. 150. Véase también ODdo, Alexandra, Vers un refranero diachronique : analyse linguistique de l'évolution des proverbes espagnols depuis le Moyen Âge, Limoges, Lambert Lucas, 2013, pp. 97-101 y 197-204.

29 Véase Maingueneau Dominique, y GréSILlon Almuth, «Polyphonie, proverbe et détournement ou un proverbe peut en cacher un autre», Langages, 73 (1984), pp. 111125 y Palma, Silvia, Les éléments figés de la langue, Paris, L’Harmattan, 2007, pp. 163-175.

30 Sobre las estructuras (o «moldes») más frecuentes en el refranero (artículo cero en posición frontal, relativas sin antecedente, oraciones averbales, etc.), ver GómEZ-JoRDANA, Sonia, Le Proverbe : vers une définition linguistique. Etude sémantique des proverbes français et espagnols contemporains, Paris, L'Harmattan, 2012, capítulo 2.

31 «Es posible -y los ejemplos abundan-que la forma truncada se lexicalice y permita la creación de una nueva entidad parémica, que a menudo elimina la forma completa. 
Los refranes truncados ${ }^{32}$ siguientes han sido amputados en diacronía de su primer miembro:

(7) De faré, faré nunca me pagué; más vale vn toma que dos te daré (García de Castro); Más vale un «toma» que un dos «te daré» (Doval, Junceda).

(8) Todo lo faré, mas casa con dos puertas no la guardaré (García de Castro); Casa con dos puertas, mala es de guardar (Doval, Junceda).

(9) Yo a buenas y vos a malas, no puede ser mas negro el cueruo que sus alas (Santillana); No puede ser más negro el cuervo que sus alas (Doval, Junceda).

En cambio, los refranes que vienen a continuación han sufrido la pérdida de su segundo miembro, un fenómeno por cierto mucho más corriente ${ }^{33}$.

(10) A quien Dios quiere bien, la casa le sabe y a quien mal, la casa y el hogar (García de Castro); A quien Dios bien quiere la casa le sabe (Santillana, Junceda).

(11) Antes que cases cata que fazes, que no es ñudo que assi desates (Santillana); Antes que te cases, mira lo que haces (Doval, Junceda).

(12) Tanto vales quanto has y tu auer de mas (Santillana); Tanto vales como tienes / Cuanto tienes, tanto vales (Doval, Junceda).

En los ejemplos (7), (8) y (9) llama la atención en primer lugar una pérdida de la rima consonante (las rimas faré/daré; faré/guardaré y malas/alas se pierden con la reconstrucción del enunciado), por lo que no parece, si nos fijamos en estos ejemplos, que la rima tenga más importancia que otros elementos rítmicos. Lo que sí se comprueba en cada cambio de la morfología original del refrán es que el enunciado final se amolda a los patrones métricos más representativos de sistema: isosilabismo ${ }^{34}$ (en (12), 4/4; en (8), (10)

Por ejemplo, la forma original A chacun son métier, et les vaches seront bien gardées está siendo suplantada por la forma truncada A chacun son métier (documentada 292 veces en frTenTen 12 contra solo 12 en el caso de la forma completa. Del mismo modo, la forma truncada Cada loco con su tema $($ Spanish $\mathrm{WaC}=11)$ está eliminando la forma completa Cada loco con su tema y cada lobo por su senda (Spanish WaC =2)» (nuestra traducción) (ANSCOMBRE, Jean-Claude, "Variantes, variations et figement en parémiologie», Cahiers de lexicologie, 116 (2020), pp. 15-44, p. 27).

32 Sobre los refranes truncados, véase también FASLA, Dalila, "Conmutación léxica, relaciones semánticas y truncamiento paremiológico», Paremia, 8 (1999), pp. 161164, y ODDo, Alexandra, "Phénomènes de troncature», en Jean-Claude Anscombre, Bernard Darbord y Alexandra Oddo (eds.), La parole exemplaire, cit., pp. 133-146.

${ }^{33}$ El fenómeno es también frecuente en sincronía. Véase ibid., pp. 133-146.

34 Aunque el isosilabismo no es sistemático, resulta frecuente. En un trabajo dedicado a los refranes franceses, Martin demuestra que todo tipo de combinaciones son posibles 
y (11) 6/6 y en (9) 7/7) en todos los casos menos el del ejemplo (7) - que bien podría ser considerado también como isosilábico sin la sinalefa entre «vale»y «un»- ${ }^{35}$. El elemento relevante aquí no es el abandono de una forma métrica o de una rima por otra forma sino la necesidad para el nuevo enunciado de presentar rasgos rítmicos o métricos identificables.

La observación de los refraneros desde una perspectiva diacrónica también proporciona ejemplos de la transformación inversa: algunos refranes se han alargado en el transcurso de la historia, sobre todo durante el Siglo de Oro -y seguramente por obra de los compiladores, sobre todo Correas- lo que plantea de nuevo la cuestión de la rima ${ }^{36}$ o del ritmo tras estas modificaciones estructurales:

(13) Una golondrina no faze verano (García de Castro, Santillana); Ni un dedo hace mano, ni una golondrina verano (Doval, Junceda).

(14) A otro perro con ese hueso (Santillana); A otro perro con ese hueso, que yo roído lo tengo (Junceda).

(15) Cada loco con su piedra (García de Castro); Cada loco con su tema, y cada lobo por su senda (Doval)/Cada loco con su tema, y cada llaga con su postema (Doval, Junceda).

(16) En cada tierra su vso (García de Castro, Santillana); En cada tierra, su uso, y en cada casa, su costumbre (Doval, Junceda).

(17) La pobreza non es villeza (García de Castro); Pobreza no es vileza, mas deslustra la nobleza (Doval, Junceda).

(18) Obras son querençias (Santillana); Obras son amores, que no buenas razones (Doval, Junceda).

(19) Por el dinero, bayla el perro (García de Castro, Santillana); Por dinero baila el perro, y por pan si se lo dan (Junceda); Por dinero baila el can, y por pan si se lo dan (Doval).

Con estos ejemplos se evidencia por un lado la importancia de los esquemas binarios en el refranero y por otro, la voluntad de

en segmentos inferiores o iguales a siete sílabas, aunque en dicho caso, su lectura tenderá a ajustar la lectura de cada parte para igualar la duración de cada una. Véase Martin, Philippe, «Intonation, rythme et eurythmie de locutions et proverbes français», en Jean-Claude Anscombre, Bernard Darbord y Alexandra Oddo (coords.), La parole exemplaire, cit., pp. 159-169, p. 168.

35 Esta hipótesis es además la más verosímil si se tiene en cuenta el aspecto semántico de oposición entre los numerales «un»y «dos».

${ }^{36}$ «La función estructurante del homeoteleuton es la que ocasiona las maquinaciones que se efectúan en la terminación de las dos cláusulas con vistas al logro de la rima. El ripio, combatido en el verso, es un recurso normal en el refrán. [...] La forma más elemental de este artificio consiste en la introducción de un nombre propio al final de uno de los miembros para que se aparee con el otro: «El asnillo de Caracena, mientras más andaba más ruín era» (HERnANDo CUADRADO, Luis Alberto, «Estilística del refrán», Paremia, 6 (1997), pp. 327-332, p. 328). 
crear una rima entre los dos miembros del refrán ${ }^{37}$, y esto resulta aún más obvio en los ejemplos (15) y (19), para los que los dos diccionarios contemporáneos ofrecen dos variantes. Son interesantes estas rimas en la medida en que se solicitan arcaísmos lingüísticos ('can', 'postema') para llegar a este resultado lo que subraya la importancia e incluso a veces la preeminencia de dichos rasgos fónicos en estas producciones de la lengua, sobre todo si se tienen en cuenta las dificultades de comprensión del enunciado que potencian tales procedimientos.

La evolución morfosintáctica en diacronía puede acarrear cambios en los determinantes, los diminutivos, los adverbios o los verbos por ejemplo ${ }^{38}$. En los dos ejemplos siguientes, la actualización del enunciado pasa del artículo cero en posición frontal ${ }^{39}$ al artículo definido, lo que, si no afecta el ritmo y el metro del primer refrán, sí permite llegar al isosilabismo (pasando de 3/4 a 4/4) en el segundo caso.

(20) A buen callar, llaman Sancho (García de Castro, Santillana); Al buen callar llaman Sancho (Doval, Junceda).

(21) Buey suelto bien se lame (Santillana); El buey suelto, bien se lame (Doval, Junceda)

Los determinantes demostrativos, que constan de tres series declinables (este/ese/aquel) crean posibilidades similares. Los refranes A otro perro con ese hueso y Aquél es tu amigo, que te quita de ruido nunca han sido modificados en diacronía por las aliteraciones que producen estas combinaciones y por el contrario, otros enunciados, en busca de efectos sonoros, son recogidos con determinantes distintos:

\footnotetext{
${ }^{37}$ La cuestión de la rima en los refranes plantea también, como lo subrayó Bizzarri, la de su documentación: «Si repasamos los refranes que aparecen en colecciones sapienciales del siglo XIII, tropezaremos con el inconveniente de que difícilmente utilicen el artificio de la rima. Pero sí lo encontramos aplicado en escritores posteriores, como es el caso de Alfonso Martínez de Toledo, quien provoca rimas aun por un proceso de aglutinación de refranes» (BIZZARri, Hugo O., El refranero castellano en la Edad Media, Madrid, Laberinto, colección Arcadia de las Letras, 2004, p. 32).

38 ODdo, Alexandra, Vers un refranero diachronique, cit.

${ }^{39}$ Se trata de un tipo de determinación muy frecuente en el refranero y muchos estudiosos lo consideran como un molde característico (véase GómEZ-JoRDANA, Sonia, Le Proverbe: vers une définition, cit.).
} 
(22) Aca lo ha Marta con sus pollos (Santillana); Allá se lo aia Marta kon sus pollos (Correas) $)^{40}$.

(23) Con esos poluos se fizieron esos lodos (Santillana); Kon esos lodos se hizieron esos polvos / Kon esos polvos se hizieron estos lodos; o esos lodos / De akellos polvos vienen estos lodos; o se hizieron estos lodos (Correas); De aquellos polvos vienen estos lodos (Junceda).

Con las modificaciones del léxico, por fin, se percibe claramente que, en numerosos casos, estos ajustes en diacronía se enmarcan también en un perfeccionamiento estilístico sistemático de los enunciados. Este afán lo ratifican los nombres presentes en el refranero castellano ya que a menudo sobresalen sus sonoridades o su ritmo a la hora de utilizarlos ${ }^{41}$ o de modificarlos en diacronía. La Marina de Los pollos de dueña Marina, ellos querían comer et ella dáuales agua (García de Castro) se convierte en Marta en la colección de Santillana y en María o Marta en la de Correas. Pascual y Vidal alternan en los refranes Malo es Pascual [y] nunca falta quien le faga mal (Santillana, Correas, Junceda) y Malo es vidal y nunca falta quien le haga mal (Correas). Incluso se pasa de Pedro y Domingo en Con lo que Pedro sana, Domjngo adolesçe a Sancha y Marta en Núñez ${ }^{42}$ y más tarde a Pedro y Sancho en la colección de Junceda. Y así se explica que el refrán medieval Qual es Yllana, tal casa mantiene (García de Castro) se recoja con cuatro nombres diferentes en los refraneros : Yllana, Olallia (Vallés) ${ }^{43}$, Olalla (Núñez) y Kostanza (Correas). Estas adaptaciones se dan también en los refranes con topónimos: las características presentes en Quien bueno es en su villa, bueno será en Seuilla (García de Castro) y Quien ruin es en su villa ruin es en Sevilla (Núñez, Correas), parecen poder

${ }^{40}$ Correas, Gonzalo, Vocabulario de refranes y frases proverbiales (1625), texte établi, annoté et présenté par Louis Combet, Bordeaux, Institut d'Études ibériques et ibéro-Américaines de l'Université de Bordeaux, 1967.

${ }^{41}$ Señalan esta propensión Hernando CUADRADO, Luis Alberto, «Estilística...», cit., p. 328 y Anscombre: «Pour assurer cette rime, la langue ne recule devant aucun procédé : utilisation de formes archaïques (anglais Marry in May, repent alway au lieu de always ), apocopes ( allemand Morgen Stund' hat Gold im Mund), déplacement d'accent tonique (Yo amo bien, que no amo a alguién, au lieu de alguien ), changement de genre ( No diga la boca / lo que pague la coca, au lieu de coco ). La langue va même jusqu'à inventer des mots, ainsi dans le plaisant dicton suivant $S$ 'il pleut le jour de Saint Georgeau / pas de fruit à noyaux» (ANSCOMBRE, Jean-Claude, "Parole proverbiale et structures métriques», Langages, 139 (2000), pp. 6-26, p. 17).

42 NúÑEZ, Hernán, Refranes y proverbios en romance (1555), edición crítica de Louis Combet, Julia Sevilla Muñoz, Germán Conde Tarrío y Josep Guia, Madrid, Guillermo Blázquez, 2001.

43 VALlÉs, Pedro, Libro de refranes y sentencias (1549), edición crítica de Jesús Cantera y Julia Sevilla, Madrid, Guillermo Blázquez, 2003. 
adaptarse a lugares tan diferentes como Sevilla o Castilla... Quien necio es en su villa, necio es en Castilla (Doval, Junceda).

En gran medida, y de ser posible, cuando se producen cambios en el léxico, se respeta el patrón rítmico, métrico o rímico original del refrán:

(24) En casa del herrero, cochillo mangorrero (Santillana); En casa del herrero, cuchillo de palo (Doval, Junceda).

(25) Faz bien [y] no cates a quien (Santillana); Haz bien y no mires a quién (Doval, Junceda).

La transformación del patrón, cuando surge, favorece a menudo, en cambio, una nueva estructuración más conforme a los esquemas más representativos del refranero, como se aprecia en el refrán siguiente, cuyo resultado final en los refraneros contemporáneos es una seguidilla $(5 / 7)^{44}$ con rima consonante (ladrón/perdón):

(26) Quien burla al burlador cien días gana de perdón (Seniloquium, Santillana); Quien roba a un ladrón, tiene cien años de perdón (Doval, Junceda).

Las reestructuraciones en pos de la rima o del metro a menudo se superponen en diacronía y permiten entender que estas modificaciones del léxico o de la morfosintaxis tienen siempre el mismo objetivo: crear una rima o ajustar un metro y un ritmo en los refranes (una seguidilla, un endecasílabo o un octosílabo, con rimas asonantes o consonantes por ejemplo) cuando estos carecían de él.

\section{Ritmo y variación diatópica}

La variación diatópica (o geográfica) en el ámbito de la paremiología es una realidad lingüística que se comprueba y se mide comparando el patrimonio de refranes recogido en las distintas hablas y áreas de la hispanidad. Cotejando las distintas publicaciones de colecciones de paremias puede establecerse que desde principios del siglo XX empiezan a recogerse, con un dinamismo creciente, obras dedicadas a la variación geográfica de la paremiología. Así se multiplican las categorías de compendios ya que a una paremiografía

$\overline{{ }^{44}}$ Los refranes que se amoldan al esquema de la seguidilla son muy frecuentes en el refranero castellano. Véase por ejemplo la evolución de Lo que ojo non vee, coraçón non qujebra (García de Castro) y Ojos que no veen, coraçon que no quiebra (Santillana) en las colecciones contemporáneas: Ojos que no ven, corazón que no siente (Doval, Junceda). Más ejemplos en OdDo, Alexandra, Vers un refranero..., cit., pp. 97-100 y pp. 197-201. 
de tipo panhispánico ${ }^{45}$, se suman colecciones peninsulares, panamericanas, nacionales (México, Perú, etc.) e incluso regionales (paremias navarras, extremeñas, piuranas, etc. $)^{46}$. Si bien la dimensión panhispánica de este refranero ha sido señalada ${ }^{47}$ por ejemplo por Torres Torres: «En su difusión americana, los refranes hispánicos, algunos muy antiguos, conservan con frecuencia la forma básica original. Ocurre, por ejemplo, con Dime con quién andas y te diré quién eres, Más vale pájaro en mano que ciento/cien volando, Al que madruga Dios le ayuda» ${ }^{48}$. Cabe puntualizar que el refranero en Latinoamérica presenta también casos interesantes de «reformulaciones del caudal heredado o nuevas acuñaciones en todo el territorio del Nuevo Mundo» ${ }^{49}$, como pueden serlo por ejemplo $E l$ mejor puerco se come la mejor guayab $a^{50}$, reformulación -o adaptación al contexto geográfico-del original El mejor puerco se come la mejor bellota. Y así recogía Kany, en su introducción a la Semántica hispanoamericana, llamativas variantes de refranes:

Más es el ruido que las nueces se ha transformado en más es la bulla que la cabuya (Ven), es más la bulla que las mazorcas (Col), más espuma que chocolate (Ant), más son las hojas que los tamales o son más hojas que almuerzo (Perú, Col, CA); más vale pan con amor que gallina con dolor y contigo pan y cebolla se han transformado en más vale atole con risas que chocolate con lágrimas y contigo la milpa es rancho y el atole champurrao [mezcla hecha con atole, chocolate y azúcar] (Méj); mientras el gato no está, los ratones bailan se ha convertido en mientras los gatos duermen, los pericotes [ratas] se pasean (Arg. Perú) ${ }^{51}$.

$\overline{45}$ «Hay diferencias, pero predominan las semejanzas. Se ha calculado que alrededor de $80 \%$ de los refranes hispanoamericanos son de origen español» (SEvilla MuÑoz, Julia, (entrevista), «Shirley L. Arora y la paremiología hispanoamericana», Paremia, 9 (2000), pp. 7-14, p. 10).

46 En lo que se refiere a los refraneros regionales publicados en la península, véase LóPEZ, Antonia, «Le proverbe et sa diversité d'usage sur le territoire espagnol», HispanismeS, 12 (2019), pp. 53-64 y «La géolinguistique au travers du prisme des proverbes», Archipélies, 8 (2019) [https://www.archipelies.org/589].

${ }^{47}$ Cabe señalar que la variación en este ámbito es un fenómeno difícil de abarcar porque a menudo se da en contextos orales.

48 Torres Torres, Antonio, «Paremiología española e hispanoamericana. Los americanismos parémicos», Dialectología, 10 (2013), pp. 87-105, p. 96.

49 Limitaremos nuestro trabajo a las variantes, o «reformulaciones del caudal heredado» (ibid., p. 87), pero son interesantes también los refranes que se fundamentan en préstamos externos al náhuatl como por ejemplo: acocote, guaje, pulque y tlachiquero en los refranes Al maguey que no da pulque, no hay que llevar acocote y $A$ acocote nuevo, tlachiquero viejo. Véase Navarro Brotons, María Lucía, «Traduire la langue, traduire la culture. Le cas des proverbes multiculturels mexicains ayant des voix du náhuatl», Synergies Tunisie, 3 (2011), pp. 125-135.

50 TORRes Torres, Antonio, «Paremiología española...», cit., p. 100.

${ }^{51}$ Kany, Charles E, Semántica hispanoamericana, Madrid, Aguilar, 1962, pp. 10-11. 
Estos enunciados presentan, a pesar de las variaciones que se observan, un significante muy similar, y como lo explicó Anscombre, no son refranes diferentes, sino una sola paremia ${ }^{52}$ y su especificidad es que su uso se limita preferentemente a algunas regiones de la hispanidad. El refrán Donde hay patrón no manda marinero (Doval, Junceda) se conoce en otros contextos geográficos como Donde manda el caporal, no gobiernan los vaqueros, y así lo explica Pérez Martínez en su Refranero mexicano: «el Refrán originado en el medio ranchero que en sentido literal significa lo que denuncia y que en sentido paremiológico, en cambio, se usa para justificar un cambio de planes a raíz de una orden jerárquicamente superior ${ }^{53}$. Para caracterizar esta multiplicación de enunciados Torres Torres cita a Guerra Garrido que en 1997 evocaba un «paralelismo paremiológico de las dos orillas de nuestro idioma»y añade unos ejemplos interesantes al corpus ${ }^{54}$ :

(27) A cada chancho le llega su San Martín ${ }^{55}$.

(28a) El chango, aunque lo vistan de seda, chango se queda ${ }^{56}$.

(29a) A falta de pan, buenas son tortillas ${ }^{57}$.

El primer ejemplo (27) conserva obviamente todos los elementos del patrón rítmico original gracias a una sencilla conmutación del léxico presente en el enunciado: chancho/puerco (dos bisílabos agudos). Este es un tipo de variación sincrónica muy frecuente en el refranero hispánico, $y$, como es precisamente en el aspecto léxico en el que se multiplican las especificidades de las variedades del español, el refranero adquiere, gracias a estas sustituciones, una actualización más dialectal de sus variantes, sin alteración del patrón rítmico (bellota/guayaba, puerco/chancho, etc.).

52 «Un ensemble de formes parémiques (dans une langue donnée, en synchronie), sera une famille parémique si : a) Les formes sont considérées comme synonymes, i.e. remplissent la même fonction parémique dans le discours ; b) Chaque membre est une variante (stricte ou large) des autres membres. Une famille parémique représente donc au fond une seule et unique parémie» (ANSCOMBRE, Jean-Claude, «Matrices rythmiques...», cit., pp. 154-155).

53 Pérez Martínez, Herón, Refranero mexicano, México, Academia Mexicana, 2004, pp. 115-116.

54 TORRes Torres, Antonio, «Paremiología española...», cit., p. 97.

${ }_{55} \mathrm{La}$ variante peninsular que recogen los refraneros es A cada puerco le llega su San Martín (Junceda).

${ }^{56}$ La variante peninsular que recogen los refraneros es Aunque la mona se vista de seda, mona se queda (Junceda).

${ }^{57}$ La variante peninsular que recogen los refraneros es A falta de pan, buenas son tortas (Junceda). 
[L]os varios ejemplos de refranes hispánicos aquí reelaborados a base del empleo sustituto de las voces de la tierra -particularmente indigenismos heredados del sustrato arahuaco taíno de las Antillas- en lugar de los términos presentes en la factura castellana primaria : Dos jueyes machos no caben en la misma cueva (cfr. en España, según Correas: Dos aves de rapiña no mantienen compañia ${ }^{58}$.

De los ejemplos (28a) y (29a) se encuentran más variantes, por ejemplo en el Refranero mexicano o en el Refranero multilingüe que recoge el Centro Virtual Cervantes, y muestran otro tipo de alteración ya que no conservan la estructura sintáctica, rímica, rítmica o métrica original. A pesar de la tematización de El chango y El mono que se produce en (28a) y en (28b) y la consiguiente reorganización sintáctica del enunciado, el cómputo métrico es similar en las tres primeras variantes (16). El nuevo patrón rítmico al que se amoldan (28a) y (28b) es una construcción tan esmerada y eficaz - por los grupos rítmicos y los paralelismos que muestra, así como por la conservación de la rima interna (seda/queda) - como la de la variante peninsular.

(28) Aunque la mona/ se vista de seda, /mona se queda (5/6/5).

(28a) El chango/, aunque lo vistan de seda, /chango se queda (3/8/5).

(28b) El mono/ aunque se vista de seda, /mono se queda, Bolivia, $(3 / 8 / 5)^{59}$.

El refrán del ejemplo (29) es interesante porque permite añadir la coordenada diacrónica a la coordenada geográfica. El refrán se documentó en etapas anteriores de la lengua con la forma $A$ mengua de pan, buenas son tortas, y con el paso del tiempo se modificó dejando paso a la variante actual (29), que, a pesar del cambio léxico, no presenta ninguna variación, ni en su patrón rítmico ni en su significado, es decir, que uno debe conformarse con lo que tiene cuando carece de algo mejor ${ }^{60}$. Y en la metáfora que permite acceder al sentido genérico del refrán se halla precisamente la clave de su variación geográfica, ya que en América la pareja pan/torta (pan de calidad menor) evoluciona en varias asociaciones léxicas que ponen de relieve las diferencias culturales y lingüísticas de cada región:

\footnotetext{
${ }^{58}$ Alvarez Nazario, Manuel, El habla campesina del país: orígenes y desarrollo del español en Puerto Rico, Río Piedras, P.R., Editorial de la Universidad de Puerto Rico, 1990, p. 455.

59 Véase el Refranero Multilingüe [https://cvc.cervantes.es/lengua/refranero/ficha.aspx?Par=58258\&Lng=0] (consultado el 29/08/2020).

${ }^{60}$ JuncedA, Luis, Diccionario..., cit., p. 9.
} 
(29) A falta de pan, buenas son tortas (6/5).

(29a) A falta de pan, buenas son tortillas (6/6).

(29b) A falta de pan, tortillas ${ }^{61}$, cemitas/cemas/semas (California, Guatemala, México, Nicaragua), casabe (Cuba, Rep. Dominicana, Venezuela) $)^{62},(5 / 3$ o $5 / 2)$.

El refrán peninsular $A$ falta de pan/buenas son tortas es un endecasílabo que se convierte, tras «cruzar el charco» en un heptasílabo en el que se utilizan cemas, y sobre todo, en un octosílabo en las versiones con tortillas, cemitas y casabe de (29a) y (29b) -uno de los patrones más frecuentes del refranero hispánico ${ }^{63}$.

\section{Ritmo y manipulaciones de refranes}

Para completar este breve panorama sobre el impacto de la variación sobre el ritmo de los refranes, queda por valorar un tipo de alteración, el que potencian las manipulaciones que se dan en el ámbito social, político o publicitario con eslóganes creados a partir de refranes. Ya evocamos al principio de este trabajo la importancia de la conservación de las principales características del enunciado original (lexía memorizada y ecónimo) ${ }^{64}$ para que opere el reconocimiento de la lexía de origen. En un trabajo dedicado a los refranes, Maingueneau también pone de manifiesto esta necesaria conservación para trasladar al nuevo enunciado la autoridad que se atribuye a los refranes. Así define el lingüista esta manipulación (o desautomatización): «[le détournement] consiste à produire un énoncé possédant les marques linguistiques de l'enonciation proverbiale mais qui n'appartient pas au stock des proverbes reconnus ${ }^{65}$.

Indagando acerca de este fenómeno en el ámbito de la publicidad, Grunig también pudo fijarse en la importancia del ritmo y de la conservación de la escansión para la reutilización de las fórmulas fijadas ${ }^{66}$. Para Martin, en francés, la estructura prosódica del refrán y la jerarquía de los acentos que lo componen es determinante

${ }_{61}$ Pérez Martínez, Herón, Refranero mexicano, Academia mexicana, 2004, p. 205.

62 Véase el Refranero Multilingüe [https://cvc.cervantes.es/lengua/refranero/ficha.aspx?Par=58064\&Lng=0] (consultado el 29/08/2020).

${ }^{63}$ DARBORD, Bernard, «La rhétorique...», cit., p. 174.

${ }^{64}$ PotTier, Bernard, Semántica general, cit., p. 20.

65 «[La manipulación] consiste en producir un enunciado que posee las marcas lingüísticas del enunciado proverbial pero que no pertenece al repertorio de refranes conocidos» (nuestra traducción) (MAIGUENEAU Dominique, y GrÉSILlon Almuth, «Polyphonie...», cit., p. 114).

${ }^{66}$ Grunig, Blanche-Noëlle, Les mots de la publicité. L'architecture du slogan, Paris, CNRS Editions, 1998, pp. 122-123. 
para su lectura ${ }^{67}$, porque el idioma francés carece precisamente de acento léxico y la acentuación de los enunciados es libre - con una limitación a grupos de siete sílabas ${ }^{68}$. Y, de hecho, aunque en español el acento léxico sí sea predominante y fijo, la observación de un corpus de refranes manipulados apunta a que las manipulaciones conservan también los acentos del modelo original, lo que obviamente favorece la identificación de la lexía memorizada y su consiguiente reconocimiento. Los ejemplos de manipulaciones publicitarias abundan ${ }^{69} \mathrm{y}$ en varias páginas de internet se han creado foros que recogen refranes manipulados a raíz de las crisis sociales y económicas (y más recientemente sanitarias) ${ }^{70}$ que asolan el país. El análisis de los refranes recogidos en los llamados «refraneros de la crisis ${ }^{71}$ permite deslindar un primer grupo de refranes que conservan todas las características (rítmicas, rímicas y métricas) de los originales al manipular solo el léxico, y otro que presenta manipulaciones más importantes:

(30) Piensa el borbón que todos son de su condición [ladrón]

En Bankia cerrada no entran moscas [boca]

En casa del herrero cartilla del paro [cuchillo de palo]

Aunque la Merkel vista de seda, Merkel se queda [mona/mona]

Al mal tiempo, mucha cara [buena]

Sobre gastos no hay nada escrito [gustos]

Por robar nada se pierde [probar]

A la cama no te irás sin deber un poco más [saber]

En martes... ni te cases ni te «embanquies» [embarques]

En el primer grupo (30), la modificación que afecta al léxico (y el consiguiente cambio semántico que conlleva) reproduce

${ }^{67}$ Martin, Philippe, «L'intonation des proverbes», Scolia, 31 (2017), pp. 119-132, p. 120.

${ }_{68}^{6}$ Martin, Philippe, «Intonation...», cit.

${ }^{69}$ Véase Ferraz Martínez, Antonio, El lenguaje de la publicidad, Madrid, Arco Libros, 2000, y ODDo, Alexandra, «Économie des slogans: de l'échonymie aux matrices rythmiques», en Irmtraud Behr \& Florence Lefeuvre (eds.), Le genre bref. Des contraintes grammaticales, lexicales et énonciatives à une exploitation ludique et esthétique, coll. Sprachwissenschaft/Linguistique, Berlin, Frank \& Timme, 2019, pp. 193-208.

70 Durante la epidemia de COVID-19 aparecieron en internet numerosas manipulaciones de refranes: Hasta el cuarenta de mayo, no visites al yayo; En abril, contagios mil; Dios los cría y ellos se contagian, etc. [https://as.com/epik/2020/04/15/portada/1586973453_647612.html].

71 Todos estos refranes se recogen en «Nuevo refranero español por la crisis» [https:// www.meneame.net/c/11512500 et ebook.com/notes/victor-hugo-saez/nuevo-refranero-español-por-la-crisis/10151824433749237/] (consultado el 25/08/2020) y en Foro-ciudad.com [https://www.foro-ciudad.com/asturias/serin/mensaje-11077050.html] (consultado el 25/08/2020). 
escrupulosamente el patrón rítmico de la lexía memorizada. Se respetan así la métrica, las rimas y los acentos del modelo con las sustituciones de ladrón/borbón; mucha/buena. El acierto de las formulaciones proviene obviamente del parecido fonográfico de los elementos manipulados, llegando incluso a crearse juegos de sonoridades asombrosas: bankia/boca; gastos/gustos; robar/probar y cuchillo de palo/cartilla de paro.

La alteración también puede ser más profunda. De ser así, el eslogan suele amoldarse a los patrones rítmicos más frecuentes del refranero.

Octosílabos:

(31) En abril recortes mil

La avaricia rompe España

Vótame y dime tonto

Dame paro y dime tonto

No dejes para Merkel / lo que pueda hacer Rajoy

Quien con deudas se acuesta, / desahuciado se levanta

Endecasílabos:

(32) Dios los cría y el gobierno los junta

No hay mal que por el INEM ${ }^{72}$ no venga

A Dios rogando y por culo dando

Quien no llora no coge subvenciones

Tanto va el cántaro a la fuente, / que al final habrá que privatizarlo

Seguidillas:

(33) Ojos que no ven, impuestos que te ponen

Ojos que no ven, corrupción evidente

Isosilabismo:

(34) Más vale euro en mano, que ciento en el Banco (6/6 o 7/7)

Obviamente, no todos los ejemplos del corpus presentan estas características, pero estas sí que pueden resultar determinantes para su identificación -y hasta su inclusión- en la categoría de los refranes. Como explica Maingueneau, en efecto, la conservación de los rasgos fónicos y rítmicos del refrán original favorece también la conservación de una característica esencial de los refranes, su

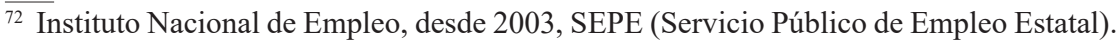


sentido genérico. Ya que una de las propiedades lingüísticas fundamentales de los refranes es la de ser frases genéricas que evocan un principio general y atemporal para caracterizar una situación gracias a un estereotipo ${ }^{73}$, esta propiedad puede verse trasladada a los ecónimos que se adaptan a su modelo tanto en el plano del significante como del significado.

Ainsi en remplaçant peine dans Eq : A chaque jour suffit sa peine par plaine, laine, naine, scène..., on produit des Ej dont la distance phonique à Eo est minimale (un seul phonème), mais qui n'entretiennent pas nécessairement une relation sémantique nette avec Eq. [...] En règle générale un détournement apparaîtra d'autant plus réussi que la modification apportée au proverbe originel aura été minimale :

- Au niveau des conditions d'emploi : une captation sera d'autant plus réussie qu'Ej sera proche des vérités véhiculées habituellement par les proverbes. Une bonne subversion, en revanche, devra exhiber avec le plus de netteté possible une contradiction entre les vérités proverbiales usuelles et le contenu de Ej.

- Au niveau du signifiant : plus la similitude entre Eo et ILi (dans la syntaxe comme dans le signifiant) sera grande, plus fort sera l'effet obtenu $^{74}$.

Estos principios explican por ejemplo lo acertado de la fórmula No hay mal que por el INEM no venga, que gracias a la conservación de su patrón rítmico y sintáctico, contribuye a cuestionar con más fuerza la verdad genérica que reza el refrán No hay mal que por bien no venga. Bien podrían explicar también el éxito de algunas de estas manipulaciones, que acaban, si los comparte una comunidad hablante, sumándose al caudal de refranes de una

73 «De hecho, hay un amplio consenso en que las paremias denotan verdades universales. Son gnómicas, y la mayoría de los estudiosos del tema consideran la gnomicidad de las paremias como de un tipo afín a la genericidad de enunciados como Los gatos cazan ratones o Los incendios destruyen los bosques» (ANSCOMBRE, Jean-Claude, «Gnomicidad/genericidad de las paremias desde el punto de vista del tiempo y del aspecto», Rilce, Revista de Filología Hispánica, 34/2 (2018), pp. 573-604, p. 132).

${ }^{74}$ «Así, al sustituir peine en Eq: À chaque jour suffit sa peine por plaine, laine, naine, scè$n e . .$, , se producen $\mathrm{Ej}$ cuya distancia fónica con Eo es mínima (un solo fonema) aunque no tengan necesariamente una relación semántica neta con Eq. [...] Generalmente, una manipulación será tanto más notable cuanto más leve sea la modificación del original: - En lo que se refiere a condiciones de empleo: una captación será tanto más notable cuanto más afinidades tenga con los valores habitualmente asociados a los refranes. Una buena subversión, en cambio, establecerá una contradicción manifiesta con las enseñanzas usuales del refranero y el contenido de Ej.

- En lo que se refiere al significante, cuanto más lograda sea la similitud entre Eo y ILi (tanto en la sintaxis como en el significado), mejor será el efecto producido» (nuestra traducción) (MAIGUENEAU Dominique, y GRÉSILlon Almuth, «Polyphonie...», cit., p. 115-116). 
nación. Candidatas ideales: Más vale euro en mano, que ciento volando; Al mal tiempo mucha cara, que podrían, en un futuro no muy lejano, ser de uso frecuente, como los enunciados franceses On ne peut pas avoir Uber et l'argent d'Uber/vouloir Uber et l'argent d'Uber, que aparece con cada vez más frecuencia en la prensa francesa:

Car pour le reste, le modèle économique est encore friable : selon un document présenté aux investisseurs, la société californienne ne dégagerait que 415 millions de dollars de chiffre d'affaires (20\% du montant de chaque course), et accuse encore 470 millions de pertes. Moralité : on ne peut pas avoir Uber et l'argent d'Uber... ${ }^{75}$

La secrétaire d'État veut Uber et l'argent d'Uber ${ }^{76}$.

Buena prueba de ello son las locuciones manipuladas que suplantan, en un momento dado, a la lexía original. Sobre el particular el caso de la locución francesa Fier comme Artaban es notable ya que poco a poco, desaparece a favor de una manipulación de gran parecido con el original. A raíz de su desautomatización en la obra de Frédéric Dard, la popularizan bajo una nueva forma (Fier comme un bar-tabac e incluso comme un p'tit banc o comme d'Artagnan) los cómicos Fernand Raynaud y más tarde Coluche. También es llamativa en castellano la fórmula de uso coloquial: Ante la duda, la más tetuda bimembre, isosilábica y con rima en $u d a . .$.

\section{Conclusiones}

La diatopía y la diacronía son dos coordenadas de la variación que afectan el enunciado parémico adaptándolo ora a la evolución histórica de la lengua, ora a las distintas variedades del habla que existen en la extensa área de uso del español. Los numerosos ejemplos sacados a colación durante este trabajo muestran a las claras que todas las transformaciones que conlleva la variación, sea diacrónica o diatópica, siempre van estrechamente ligadas a la métrica, a la rima y al ritmo y que el ritmo con frecuencia ocupa un lugar decisivo no solo en la acuñación de los refranes, sino también en su conservación y en su evolución. Y es que, como lo habían señalado

\footnotetext{
${ }^{75}$ Manipulación del refrán francés On ne peut pas avoir le beurre et l'argent du beurre (de sentido equivalente a 'no se puede estar en misa y repicando') en la página del periódico La tribuna [https://www.latribune.fr/opinions/uber-et-l-argent-d-uber-488829. html] (consultado el 16/12/2019).

76 L'express [https://blogs.lexpress.fr/nos-vies-numeriques/2014/09/30/la-secretaire-detat-veut-uber-et-largent-duber/] (consultado el 2/09/2020).
} 
Dessons y Meschonnic ${ }^{77}$, y más recientemente Martin, los patrones rítmicos de los refranes son en sí otro componente más para acceder al sentido y a la iconicidad de dichos enunciados:

Ainsi, la locution 2/5 La nuit / tous les chats sont gris est prononcée avec un ralentissement de débit syllabique sur le premier groupe accentuel la nuit, et avec une accélération sur le groupe tous les chats sont gris. Il en va de même pour le proverbe 3/6 Fleur flétrie / jamais ne refleurit, avec fleur flétrie ralenti et jamais ne refleurit accéléré. Ces variations de débit sont susceptibles de déclencher une interprétation iconique, invoquant l'agilité et la souplesse de déplacement des chats en pleine nuit, ou la lenteur attachée au flétrissement des fleurs ${ }^{78}$.

Este patrón rítmico estructurante y los artificios rítmicos propios de estas producciones son a la vez factores de cambio y de estabilidad del refranero $\mathrm{y}$, en cierto modo, regulan las posibilidades de transformación del enunciado e incluso sus potenciales manipulaciones. Como explicar si no la conservación de arcaísmos en nuestro refranero contemporáneo (A donde fueres haz lo que vieres; Quien lengua ha a Roma va), pero también su corrección (Haz bien y no mires a quien (1)) o la actualización dialectal de las variantes de A falta de pan, tortillas (cemitas, /casabe).

77 Dessons, Gérard, y Meschonnic, Henri, Traité du rythme. Des vers et des proses, París, Nathan/VUEF, 2003, p. 122.

78 «Así, la locución 2/5 La nuit / tous les chats sont gris se pronuncia ajustando el habla (disminución del flujo) para crear un primer grupo acentual en La nuit, y otro (aceleración del flujo) en tous les chats sont gris. Pasa lo mismo con el refrán 3/6 Fleur flétrie / jamais ne refleurit, con fleur flétrie más lento y jamais ne refleurit acelerado. Estas variaciones del flujo pueden engendrar una interpretación icónica: la de la agilidad y flexibilidad de los felinos al desplazarse en plena noche, o la lentitud que se asocia con las flores que se marchitan», MARTIN, Philippe, «Intonation...», cit., p. 169. 